\title{
Impact of carotid stenosis on cerebral hemodynamic failure and cognitive impairment progression: a narrative review
}

\author{
Giovanna Viticchi $^{1} \wedge$, Lorenzo Falsetti ${ }^{2}$, Eleonora Potente ${ }^{1}$, Marco Bartolini $^{1}$, Mauro Silvestrini $^{1}$ \\ ${ }^{1}$ Neurological Clinic, Marche Polytechnic University, Ancona, Italy; ${ }^{2}$ Internal and Subintensive Medicine, Ospedali Riuniti Ancona, Italy \\ Contributions: (I) Conception and design: G Viticchi, L Falsetti; (II) Administrative support: M Bartolini, M Silvestrini; (III) Provision of study \\ materials or patients: E Potente; (IV) Collection and assembly of data: G Viticchi, M Silvestrini; (V) Data analysis and interpretation: L Falsetti; \\ (VI) Manuscript writing: All authors; (VII) Final approval of manuscript: All authors. \\ Correspondence to: Giovanna Viticchi. Neurological Clinic, Marche Polytechnic University, Via Conca 1, 60020 Ancona, Italy. Email: viticchi.g@gmail.com.
}

\begin{abstract}
Carotid atherosclerosis has a relevant impact on cerebral blood flow regulation. There is accruing evidence that hemodynamic impairment related to the presence of a significant carotid lumen narrowing may predispose to the development of cerebral dysfunctions, including a reduction in cognitive abilities. In the last years an increasing number of findings showed that carotid stenosis did contribute to cognitive impairment not only in relation to the occurrence of cerebral ischemic lesions, but also as an independent risk factor. The principal mechanisms involved are chronic hypoperfusion, microembolization and cerebrovascular reactivity impairment. Moreover, more recent studies showed alterations of regional functional connectivity. In this narrative review, we analyzed the relationships between carotid stenosis, cerebral hemodynamic derangement and cognitive impairment onset and progression, and underlined that cognitive impairment is the final result of the complex interaction between different elements, including also collateral circulation, cerebral hemodynamic status, brain connectivity and pro-inflammatory state. Further, therapeutic approaches, with a specific focus on vascular risk factors correction and on the effectiveness of surgical or endovascular interventions were discussed. We particularly focused our attention on the concept of "asymptomatic carotid stenosis", and how could a cognitive impairment improve after an intervention, and how this could change the indications to surgical approach. Larger studies and randomized controlled trials are urgently required to better define time, characteristics and effectiveness of both medical and surgical/ endovascular approaches.
\end{abstract}

Keywords: Carotid stenosis; cognitive impairment; cerebral hemodynamic; dementia

Submitted Oct 31, 2020. Accepted for publication Mar 05, 2021.

doi: 10.21037/atm-20-7226

View this article at: http://dx.doi.org/10.21037/atm-20-7226

\section{Introduction}

Carotid stenosis has been traditionally considered as a main determinant for an increased risk of ischemic cerebrovascular events through thromboembolic and hemodynamic mechanisms (1).

During the last twenty years, increasing evidence emphasized the narrow relationship between carotid stenosis and dementia. Particularly, the association between cerebral hemodynamic derangement and cognitive impairment has strengthened the concept that vascular dementia and Alzheimer's disease (AD), initially considered as completely different entities, could be the expression of common pathophysiological mechanisms. This hypothesis was initially underlined in neuropathological studies. In

\footnotetext{
$\wedge$ ORCID: 0000-0002-1799-1563.
} 
the "Nun Study", amyloid angiopathy and periventricular white matter lesions were found both in $\mathrm{AD}$ and in vascular dementia patients (2). Recent findings confirmed a large prevalence of both atherosclerosis and arteriolosclerosis in patients with $\mathrm{AD}(3,4)$ and found a correlation between cerebral atherosclerosis and the typical neuropathological aspects of degenerative dementias, including neuritic plaques, neurofibrillary tangles and cerebral amyloid angiopathy, (5). Further, a narrow relationship between $\mathrm{AD}$ and vascular derangement has been described $(6,7)$. One of the most investigated aspects about this issue was the role of carotid stenosis in dementia onset and progression (8).

In this paper, we reviewed the most recent literature on this topic in an attempt to contribute to a better definition of the pathophysiologic correlations between carotid stenosis and cognitive impairment occurrence. Further, we discussed on possible effects of different therapeutic approaches, particularly the management of vascular risk factors and the stenosis correction with surgical and endovascular procedures.

We present the following article in accordance with the Narrative Review checking checklist (available at http:// dx.doi.org/10.21037/atm-20-7226).

\section{Methods}

We searched PubMed/Medline for case reports, reviews and original research articles in English language in the timeframe from January $1^{\text {st }}, 1990$ to October $1^{\text {st }}$, 2020. The group of reviewers favoured the inclusion of papers from the past 10 years, although they did not exclude highly referenced older reports; the reference lists of articles identified by this search strategy was also reviewed, and the working group selected those references judged to be relevant. We used MeSH major terms and considered: "carotid stenosis" $[\mathrm{MeSH}]$ or "cognitive impairment" $[\mathrm{MeSH}]$ or "dementia" $[\mathrm{MeSH}]$ or "hemodynamic" $[\mathrm{MeSH}]$ or "blood flow, regional" $[\mathrm{MeSH}]$ or "circulation, collateral blood" $[\mathrm{MeSH}]$, or "doppler ultrasound imaging" $[\mathrm{MeSH}]$ or "doppler sonography, transcranial" $[\mathrm{MeSH}]$, alone or in combination.

\section{Part I: association between carotid stenosis and cognitive impairment}

\section{Pathophysiological and epidemiological correlates}

The pathological aspects of degenerative dementias are traditionally referred to amyloid plaques and fibrillary tangles deposition. On the other hand, the presence of cerebral vascular impairment is considered a marker of vascular dementia that is mainly characterized by a compromise of subcortical cognitive functions. During the last years, however, increasing evidences suggested an association between cerebrovascular diseases and $\mathrm{AD}$ onset and progression. More interestingly, some studies investigated the possible role of carotid stenosis in cognitive impairment. In this respect, the evaluation of cerebral hemodynamics seems to have a role in $\mathrm{AD}$ patients' assessment $(6,7)$. The first studies about this topic (9-11) showed a possible link between the two pathological entities. The results, based on small cohorts of patients suggested that, in the presence of a significant carotid stenosis, AD patients had worse performances at neuropsychological evaluations when compared to patients without vessel impairment.

It has been postulated that the extent of brain vascular derangement, considering both carotid atherosclerosis and the amount of vascular risk factors, can significantly influence the severity of cognitive impairment $(6,12)$. For this reason, the opportunity to introduce carotid artery ultrasound and echocardiography in the diagnostic process for the assessment of subjects at risk of developing cognitive impairment has been recommended (13).

Several studies investigated the role of carotid lumen narrowing on cognitive deterioration, suggesting that a significant stenosis could be considered as an independent risk factor for every type of dementia, including $\mathrm{AD}$ (14-16).

The Tromsø Study (17) supported relevant data on large cohort of patients with cognitive impairment. By using an extensive neuropsychological assessment, a more serious reduction in cognitive performances was detected among patients with asymptomatic carotid stenosis when compared to the control group. Further, the presence of carotid stenosis was a risk factor for cognitive impairment even in patients without vascular lesions at brain MRI (17). This last point can be considered as very relevant because previous studies failed to support specific data on the impact of cerebral vascular lesions so suggesting that carotid stenosis could be interpreted as an expression of general atherosclerosis and not as a direct cause of dementia (18). On the contrary, the results of the Tromsø Study confirmed the concept that carotid stenosis can be considered as an independent risk factor for cognitive impairment (17). Further, a relationship between severity of stenosis and extent of cognitive deterioration was found. This association was also confirmed by the results of The Cardiovascular 
Health Study which included more than 4,000 patients (19). A sub-study of the Framingham project, the Framingham Offspring Study, specifically evaluated this topic. A sample of 1,971 subjects was evaluated by neuropsychological tests, carotid ultrasound and MRI. The presence of carotid stenosis was significantly associated to poorer cognitive performances (20).

\section{Possible pathogenetic mechanisms}

The contribution of carotid stenosis to cognitive impairment has been traditionally considered as specifically related to the occurrence of cerebral ischemic lesions. Under this light, carotid stenosis has been viewed as a "marker" of brain atherosclerosis subsequent to the negative effects of vascular risk factors $(14,18)$. Against this concept, several studies showed that carotid stenosis can be an independent risk factor for cognitive deterioration, suggesting its prominent role in the progression of dementia $(18,19)$. In the last twenty years, several authors investigated the role of asymptomatic stenosis, in order to identify candidate risk factors for pre-symptomatic dementia. Hypertension, diabetes, dyslipidemia, smoking and other conditions, including sleep related breathing disorders, predisposing to vascular diseases, are considered as significant risk factors for both carotid stenosis and several subtypes of dementia. The possible pathogenetic mechanism involves the induction of structural damages at the level of brain and vascular structures, with the final result of global cerebral dysfunction and cerebrovascular impairment (6,21). Several studies found that vascular risk factors have a significant impact on the onset (22-26) and the progression of cognitive impairment (27-29). The negative impact of carotid stenosis on cognitive efficiency may occur with different modalities. The reduction of cerebral perfusion pressure can induce a chronic hypoperfusion (21,30,31). Hypoperfusion was present in a large part of patients with carotid stenosis, and this fact was demonstrated also by perfusion-weighted magnetic resonance imaging (32).

The collateral circulation has the function to maintain an adequate blood flow also in the case of acute vessel occlusion, and it is fundamental for the penumbra recovery during ischemic stroke. In chronic stenosis, the activation of collateral vessels can contribute to contrast hypoperfusion. This could explain the fact that the degree of stenosis is not always associated with the presence of hypoperfusion (33).

Recently, several studies showed, with the use of MRI or digital subtraction angiography, the relevance of collateral circles in preserving cognitive functions in patients with carotid stenosis (34). In the presence of a failure of the collateral vessels system, blood flow derangement occurs and it can induce a worsening of cerebral functions, including cognitive activities. Moreover, the efficiency of collateral vessels can progressively deteriorate thus exposing cerebral regions supported by collateral circulation to a higher risk of developing hypoperfusion and cerebral ischemic damages or dysfunctions (35).

Moreover, carotid stenosis could contribute directly to lacunar infarcts and WMLs development (36). The main mechanism is the microembolization by unstable or ulcerated plaques (33). The evaluation of plaque characteristics is a relevant but very often neglected aspect. In the large majority of studies, carotid lumen narrowing is definite as "significant" or "non-significant". The concept of microembolization is narrowly related to the so-called "vulnerable plaques", characterized by a dominant fat component. Recent studies highlighted the correlation between cognitive impairment and physical strain in carotid atherosclerotic plaques. In particular, plaque instability has been associated to microembolization and white matter changes (37).

On the other hand, the calcified components seem to exert a protective role (38-40), though same authors found a relationship between carotid calcifications and cognitive impairment in asymptomatic carotid stenosis (41). From $15 \%$ to $19 \%$ of patients with carotid stenosis have a documented brain microembolization (42). In patients with dementia, a significantly higher extent of spontaneous microembolization with respect to controls has been demonstrated (43). Microembolization could contribute to cognitive impairment by inducing ischemic damage and micro-circle derangement. However, some studies did not find an increased prevalence of spontaneous microembolization among demented patients but confirmed the narrow relationship with carotid stenosis (44). WMLs are commonly associated with cognitive impairment. The Rotterdam study showed that lower cognitive scores characterized patients with major impact of WMLs (45). WMLs are particularly associated with carotid stenosis and tend to progressively increase (35). From a pathological point of view, WMLs and silent infarcts may be considered as a continuum. Also cerebral infarcts and microinfacts are associated with carotid stenosis, and all these entities are correlated with cognitive impairment (46). WMLs are related to a gliotic reaction due to chronic hypoperfusion while silent infarcts are the results of the occlusion of 
perforating arteries (34).

Another relevant topic about the relationships between carotid disease and dementia is represented by the role of cerebral atrophy. Carotid stenosis is a marker of cerebral atherosclerosis and can be associated to hypoperfusion as a possible determinant of cerebral atrophy (47) which is a relevant neuropathological marker of dementia (48). Further, carotid stenosis could affect cerebral hemodynamics at the microcircle level. Cerebrovascular reactivity (CVR) is a peculiar function of cerebral homeostatic mechanisms (49). In particular, it is an expression of the ability of cerebral hemodynamic mechanisms to protect the neurovascular unit (NU) from noxious stimuli as hypo-oxygenation. $\mathrm{NU}$ is a complex brain-vascular interface that regulates the interaction among endothelium, astrocytes, neurons and supporting cells (e.g., microglia and oligodendroglia). Further, it modulates brain regional blood flow (50). NU derangement could be implied in cognitive impairment by contributing to $A \beta$ plaques accumulation and to the inflammation observed in dementia patients. Vascular risk factors are the main determinants for NU derangement by inducing oxidative stress damage in different districts. Oxidative stress is associated to the release of proinflammatory cytokines and the activation of pro-apoptotic pathways with a consequent endothelial dysfunction, decreased synthesis of nitric oxide and sympathetic activation. Further, the inflammatory state is associated with activated microglia and the consequent $\mathrm{A} \beta$ deposition (51). The $\mathrm{A} \beta$ deposition, associated with impaired cerebral hemodynamic and chronic hypoperfusion, contributes to cholinergic dysfunction and neuronal loss $(13,52)$. Several studies extensively investigated this topic by using, as a marker of CVR the breath-holdingindex (BHI), an accurate and easy-to-obtain tool to evaluate NU integrity (52). The results showed that an association between carotid stenosis and impaired BHI is significantly associated with the risk of developing cognitive impairment $(29,52)$. Moreover, as previously reported, carotid stenosis can contribute to chronic hypoperfusion and cerebral circulation derangement, which increases the probability of NU dysfunction. The relationships between carotid stenosis, hemodynamic impairment and cognitive deterioration were confirmed both for unilateral and bilateral stenosis. Balucani et al. (53) demonstrated that in patients with bilateral carotid stenosis the compromise of cognitive functions is strictly related to the cerebral hemodynamic status. A specific relationship was, in fact found between the more compromised, in terms of hemodynamic impairment, hemisphere and the failure in specific cognitive performances (53).

Moreover, a reduction in CVR has been associated to an increased intima media thickness and arterial stiffness at the carotid level. In turn, carotid wall thickness is associated to chronic hypoperfusion and lower blood flow velocity, while increased stiffness is associated to the extent of WMLs (54-56).

Finally, brain-vascular dysfunction can lead to an alteration of regional functional connectivity with the result of inducing single or multiple cognitive alterations (57). Some studies showed a relevant reduction of functional connectivity in the frontoparietal network ipsilaterally to carotid stenosis and an impairment of the bilateral interhemispheric functional connectivity (16).

The possible role of inflammation as a possible mechanism for cognitive dysfunction related to carotid disease deserves consideration (54). Both atherosclerosis and amyloid deposits share a pro-inflammatory state characterized by microglial and astrocytes activation. It is possible that they have common effectors or activation ways. In this respect, the pro-inflammatory state at carotid plaques level could stimulate the production of cytokines, including inteleukin-1, that are associated to an increased risk of cognitive impairment (58). Microembolization can damage the blood-brain barrier with a consecutive activation of immunity response and a final deposition of amyloid (54). Chronic hypoperfusion could also induce a severe structural alteration of brain cells (59). All these findings are probably relevant for the possibility to individuate new targets for effective treatments.

Based on the existing evidences, it is possible to sustain that cognitive impairment is the final result of the complex interaction between different elements, traditionally including hypoperfusion, silent infarcts and WMLs. The relevance of collateral circulation and cerebral hemodynamic status, brain connectivity and pro-inflammatory state deserves further consideration. Probably, a dominant mechanism able to fully explain the increased risk of cognitive impairment in subjects with carotid stenosis does not exist. A failure in cognitive performances leading to dementia should then be considered as the results of a synergic and complex interaction among different factors able to induce unfavorable changes at the brain level (Figure 1).

\section{Neuropsychological functions impairment in carotid stenosis}

Data about the possible existence of a compromise in 


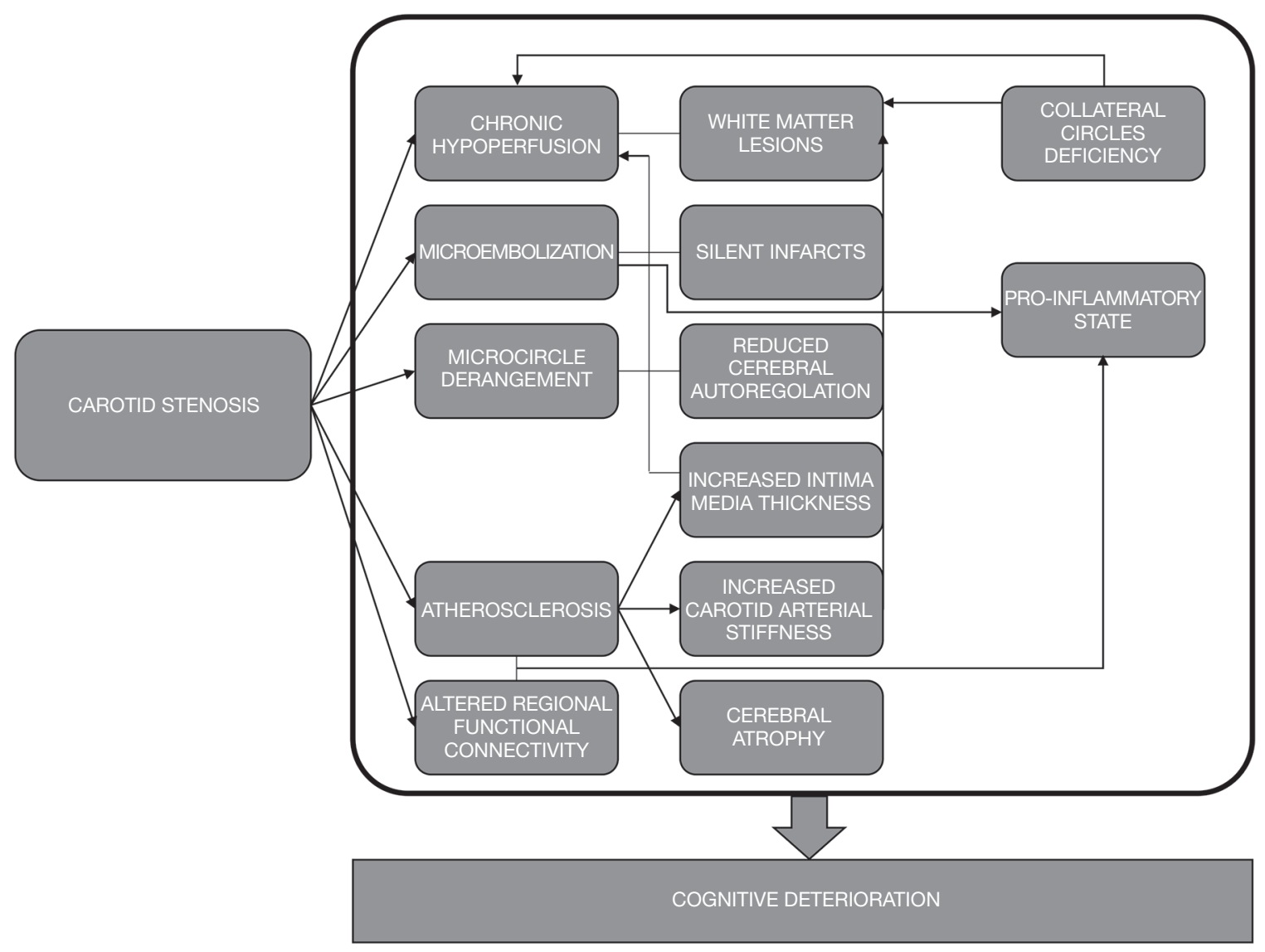

Figure 1 Proposed mechanisms for the association between carotid stenosis and cognitive impairment.

specific cognitive functions in patients with carotid stenosis do not allow to reach definitive conclusions. The results obtained are extremely variable, especially due to the lack of homogeneity in the choice of the neuropsychological test batteries. When considering a global evaluation of cognitive status, the Mini-Mental State Examination (MMSE) resulted typically impaired in patients with carotid stenosis, and the extent of scores reduction was narrowly associated with the degree of stenosis (60). Other studies employed The Montreal Cognitive Assessment (MoCA) with similar results (14).

In the Framingham Offspring Study, executive functions resulted more significantly impaired (20), while other studies showed a more relevant compromise in learning, memory or motor/processing speed $(33,54)$. Rao et al. (36) compared the different neuropsychological approaches and found that findings were too contrasting and inhomogeneous to be correctly interpreted and compared. In general, the findings about this topic, underline an impact on general cognitive functions and some more involvement of memory, attention, and executive functions but without a specific domain compromise $(34,36,54,61)$.

Several variables seem to influence the extent and characteristics of cognitive impairment. Patients with more severe stenosis usually obtain lower scores at the neuropsychological evaluation with respect to mild-tomoderate stenoses (54). This could be partially explained by the "Spencer Curve" theory: carotid stenosis can negatively affect cerebral perfusion only when the lumen narrowing is severe (62).

The plaque characteristics are also associated to the typology of cognitive functions impairment. Vulnerable plaques resulted particularly associated with a reduction of psychomotor speed, attention, and mental flexibility (63).

Finally, the side of stenosis seems to play a significant role. Different studies showed a direct correlation between the side of stenosis and the specific neuropsychological functions impairment. In particular, a failure in test exploring verbal 
functions seems to characterize patients with left stenosis. On the other hand, a compromise in visuo-spatial abilities can be found in the presence of right stenosis. It is of interest to underline that the probability to detect a lateralized cognitive dysfunction is significantly higher in the presence of an alteration of CVR, even in asymptomatic subjects (64). All these neuropsychological alterations in carotid stenosis presented temporal and spatial correlates in neural activities characteristics. A recent study employing a resting-state functional magnetic resonance showed a correlation among different cognitive tests impairment and the activity of specific brain areas (as bilateral dorsomedial prefrontal cortex and hippocampus) evaluated by the amplitude of lowfrequency fluctuations (65).

\section{Part II: effects of different treatment strategies}

The possibility to individuate correctable risk factors for cognitive impairment is considered as very important because a relevant quote of conditions involved in promoting cognitive decline are unmodifiable and not susceptible to any type of treatment (66). On the contrary, patients with carotid disease can benefit from pharmacological or interventional treatments.

Dementia, stroke and carotid stenosis share several modifiable risk factors including hypertension, diabetes, dyslipidemia and smoking. Concerning the relationship between stroke and carotid stenosis, the first therapeutic approach is based on the correction of the vascular risk profile. Recent guidelines underlined the relevance of the "best medical therapy" based on antiplatelet, antihypertensive and lipid-lowering drugs in addition to changes in lifestyle. The medical approach should be recommended as long as possible before reaching the decision to refer patients to invasive treatments for stenosis correction (67).

When the medical approaches are not considered sufficient, as in the case of patients with recent ipsilateral cerebral ischemic events or practicable, carotid endarterectomy (CEA) or carotid artery stenting (CAS) become the first-choice treatments. The possibility of an invasive approach for subjects with no history of cerebrovascular disease is particularly controversial and debated.

Several studies investigated the role of carotid stenosis surgical or endovascular correction on cognitive outcomes with a special focus on subjects without a history of cerebrovascular diseases. Most of them found a substantial improvement in cognitive functions, both after CEA and CAS. In particular, an improvement in executive and motor tests was associated with CEA (68). Studies regarding the impact of CAS showed an improvement in the global cognitive status evaluated by MMSE or MoCA $(61,69)$.

On the other hand, some studies found a worsening in cognitive functions after stenosis treatment, probably due to interventional complications, including a reduction in perfusion pressure or embolization during the procedures $(61,70)$. Other elements, like the carotid clamping or the duration of procedures, appear to be less relevant in the determination of cognitive outcomes.

From a pathophysiologic point of view, the positive effects of CEA or CAS in reducing the risk of cognitive deterioration could involve an improvement of cerebral perfusion (71). Further, the correction of a source of microembolization is expected to reduce the probability of silent infarcts. Some studies showed that improvement in CVR ipsilaterally to the stenosis correction is associated to an increase in cognitive scores even in symptomatic patients (72).

Carotid stenosis is considered asymptomatic in the absence of recent ipsilateral cerebrovascular events. The possibility to consider cognitive impairment as specifically linked to carotid stenosis, could change the prospective and approaches to patients. The unfavorable hemodynamic effects of carotid stenosis could be easily evaluated by non-invasive instrumental exams as transcranial Doppler ultrasonography. This approach has proved to be also effective to detect a threshold of arterial flow velocity changes that can predict cognitive impairment (73). This element could prove strategic in the management of carotid atherosclerosis (74). If cognitive impairment can be shown to be reversible by revascularization, then a redefinition of symptomatic carotid stenosis should be considered as a medical priority. Some insights about this topic are expected from the results of the CREST-2 study (75).

\section{Conclusions}

The preponderance of the evidence suggests an association between carotid stenosis and cognitive impairment onset and progression. Recent findings suggest that revascularization procedures should be considered as a way to restore hemodynamically-induced cognitive impairment. However, inconsistencies across studies with respect to study designs, timelines of post-procedural cognitive assessments, lack of control groups or diversity in selection, variability of neuropsychological measures 
weaken the overall findings. Larger studies and randomized controlled trials are urgently required to better define time, characteristics and effectiveness of both medical and surgical/endovascular approaches.

\section{Acknowledgments}

Funding: None.

\section{Footnote}

Provenance and Peer Review: This article was commissioned by the Guest Editor (Kosmas I. Paraskevas) for the series "Carotid Artery Stenosis and Stroke - Prevention and Treatment Part II" published in Annals of Translational Medicine. The article has undergone external peer review.

Reporting Checklist: The authors have completed the Narrative Review reporting checklist. Available at http:// dx.doi.org/10.21037/atm-20-7226

Conflicts of Interest: All authors have completed the ICMJE uniform disclosure form (available at http://dx.doi. org/10.21037/atm-20-7226). The series "Carotid Artery Stenosis and Stroke - Prevention and Treatment Part II" was commissioned by the editorial office without any funding or sponsorship. The authors have no other conflicts of interest to declare.

Ethical Statement: The authors are accountable for all aspects of the work in ensuring that questions related to the accuracy or integrity of any part of the work are appropriately investigated and resolved.

Open Access Statement: This is an Open Access article distributed in accordance with the Creative Commons Attribution-NonCommercial-NoDerivs 4.0 International License (CC BY-NC-ND 4.0), which permits the noncommercial replication and distribution of the article with the strict proviso that no changes or edits are made and the original work is properly cited (including links to both the formal publication through the relevant DOI and the license). See: https://creativecommons.org/licenses/by-nc-nd/4.0/.

\section{References}

1. Kernan WN, Ovbiagele B, Black HR, et al. Guidelines for the prevention of stroke in patients with stroke and transient ischemic attack: a guideline for healthcare professionals from the American Heart Association/ American Stroke Association. Stroke 2014;45:2160-236.

2. Snowdon DA, Greiner LH, Mortimer JA, et al. Brain infarction and the clinical expression of Alzheimer disease. The Nun Study. JAMA 1997;277:813-7.

3. Arvanitakis Z, Capuano AW, Leurgans SE, et al. Relation of cerebral vessel disease to alzheimer's disease dementia and cognitive function in older persons: a cross-sectional study. Lancet Neurol 2016;15:934-43.

4. Buratti L, Balestrini S, Altamura C, et al. Markers for the risk of progression from mild cognitive impairment to Alzheimer's disease. J Alzheimers Dis 2015;45:883-90.

5. Yarchoan M, Xie SX, Kling MA, et al. Cerebrovascular atherosclerosis correlates with Alzheimer's pathology in neurodegenerative dementias. Brain 2012;135:3749-56.

6. de la Torre JC. The vascular hypothesis of Alzheimer's disease: bench to bedside and beyond. Neurodegener Dis 2010;7:116-21.

7. Breteler MM. Vascular risk factors for Alzheimer's disease: an epidemiologic perspective. Neurobiol Aging 2000;21:153-60.

8. Viticchi G, Falsetti L, Vernieri F, et al. Vascular predictors of cognitive decline in patients with mild cognitive impairment. Neurobiol Aging 2012;33:1127.e1-9.

9. Naugle RI, Bridgers SL, Delaney RC. Neuropsychological signs of asymptomatic carotid stenosis. Arch Clin Neuropsychol 1986;1:25-30.

10. Benke T, Neussl D, Aichner F. Neuropsychological deficits in asymptomatic carotid artery stenosis. Acta Neurol Scand 1991;83:378-81.

11. Rao R. The role of carotid stenosis in vascular cognitive impairment. J Neurol Sci 2002;203-204:103-7.

12. de la Torre JC. Carotid artery ultrasound and echocardiography testing to lower the prevalence of Alzheimer's disease. J Stroke Cerebrovasc Dis 2009;18:319-28.

13. Silvestrini M, Viticchi G, Altamura C, et al. Cerebrovascular assessment for the risk prediction of Alzheimer's disease. J Alzheimers Dis 2012;32:689-98.

14. Martinić-Popović I, Lovrenčić-Huzjan A, Demarin V. Advanced asymptomatic carotid disease and cognitive impairment: an understated link? Stroke Res Treat 2012;2012:981416.

15. Buratti L, Balucani C, Viticchi G, et al. Cognitive deterioration in bilateral asymptomatic severe carotid stenosis. Stroke 2014;45:2072-7.

16. Cheng HL, Lin CJ, Soong BW, et al. Impairments 
in cognitive function and brain connectivity in severe asymptomatic carotid stenosis. Stroke 2012;43:2567-73.

17. Mathiesen EB, Waterloo K, Joakimsen O, et al. Reduced neuropsychological test performance in asymptomatic carotid stenosis: the Tromsø Study. Neurology 2004;62:695-701.

18. Sztriha LK, Nemeth D, Sefcsik T, et al. Carotid stenosis and the cognitive function. J Neurol Sci 2009;283:36-40.

19. Johnston SC, O'Meara ES, Manolio TA, et al. Cognitive impairment and decline are associated with carotid artery disease in patients without clinically evident cerebrovascular disease Ann Intern Med 2004;140:237-47.

20. Romero JR, Beiser A, Seshadri S, et al. Carotid artery atherosclerosis, MRI indices of brain ischemia, aging, and cognitive impairment: the Framingham study. Stroke 2009;40:1590-6.

21. de la Torre JC. Vascular risk factor detection and control may prevent Alzheimer's disease. Ageing Res Rev 2010;9:218-25.

22. Viticchi G, Falsetti L, Buratti L, et al. Framingham risk score can predict cognitive decline progression in Alzheimer's disease. Neurobiol Aging 2015;36:2940-5.

23. Valenti R, Pantoni L, Markus HS. Treatment of vascular risk factors in patients with a diagnosis of Alzheimer's disease: a systematic review. BMC Med 2014;12:160.

24. Lattanzi S, Viticchi G, Falsetti L, et al. Visit-to-visit blood pressure variability in Alzheimer disease. Alzheimer Dis Assoc Disord 2014;28:347-51.

25. Buratti L, Luzzi S, Petrelli C, et al. Obstructive Sleep Apnea Syndrome: An Emerging Risk Factor for Dementia. CNS Neurol Disord Drug Targets 2016;15:678-82.

26. Deschaintre Y, Richard F, Leys D, et al. Treatment of vascular risk factors is associated with slower decline in Alzheimer disease. Neurology 2009;73:674-80.

27. Viticchi G, Falsetti L, Buratti L, et al. Framingham Risk Score and the Risk of Progression from Mild Cognitive Impairment to Dementia. J Alzheimers Dis 2017;59:67-75.

28. Viticchi G, Falsetti L, Vernieri F, et al. Apolipoprotein E genotype and cerebrovascular alterations can influence conversion to dementia in patients with mild cognitive impairment. J Alzheimers Dis 2014;41:401-10.

29. Buratti L, Viticchi G, Falsetti L, et al. Vascular impairment in Alzheimer's disease: the role of obstructive sleep apnea. J Alzheimers Dis 2014;38:445-53.

30. Buratti L, Viticchi G, Falsetti L, et al. Thresholds of impaired cerebral hemodynamics that predict short-term cognitive decline in asymptomatic carotid stenosis. J Cereb Blood Flow Metab 2016;36:1804-12.
31. Derdeyn CP. Hemodynamics and oxygen extraction in chronic large artery steno-occlusive disease: Clinical applications for predicting stroke risk. J Cereb Blood Flow Metab 2018;38:1584-97.

32. Khan AA, Patel J, Desikan S, et al. Asymptomatic carotid artery stenosis is associated with cerebral hypoperfusion. J Vasc Surg. 2020;6:S0741-5214(20)32328-4.

33. Lal BK, Dux MC, Sikdar S, et al. Asymptomatic carotid stenosis is associated with cognitive impairment. J Vasc Surg 2017;66:1083-92.

34. Porcu M, Cocco L, Saloner D, et al. Extracranial Carotid Artery Stenosis: The Effects on Brain and Cognition with a Focus on Resting-State Functional Connectivity. J Neuroimaging 2020;30:736.

35. Chen YF, Kuo YS, Wu WC, et al. Association between leukoaraiosis and cerebral blood flow territory alteration in asymptomatic internal carotid artery stenosis. Clin Radiol 2018;73:502.e9-502.e14.

36. Rao $\mathrm{R}$. The role of carotid stenosis in vascular cognitive impairment. Eur Neurol 2001;46:63-9.

37. Dempsey RJ, Varghese T, Jackson DC, et al. Carotid atherosclerotic plaque instability and cognition determined by ultrasound-measured plaque strain in asymptomatic patients with significant stenosis. J Neurosurg 2018;128:111-9.

38. Saba L, Saam T, Jäger HR, et al. Imaging biomarkers of vulnerable carotid plaques for stroke risk prediction and their potential clinical implications. Lancet Neurol 2019;18:559-72.

39. Saba L, Sanfilippo R, Pascalis L, et al. Carotid artery abnormalities and leukoaraiosis in elderly patients: evaluation withMDCT. AJR Am J Roentgenol 2009; 192:W63-70.

40. Silvestrini M, Altamura C, Cerqua R, et al. Ultrasonographic markers of vascular risk in patients with asymptomatic carotid stenosis. J Cereb Blood Flow Metab 2013;33:619-24.

41. Chu Z, Cheng L, Tong Q. Carotid artery calcification score and its association with cognitive impairment. Clin Interv Aging 2019;14:167-77.

42. Brott T, Tomsick T, Feinberg W, et al. Baseline silent cerebral infarction in the Asymptomatic Carotid Atherosclerosis Study. Stroke 1994;25:1122-9.

43. Purandare N, Burns A, Daly KJ, et al. Cerebral emboli as a potential cause of Alzheimer's disease and vascular dementia: case- control study. BMJ 2006;332:1119-24.

44. Voshaar RC, Purandare N, Hardicre J, et al. Asymptomatic spontaneous cerebral emboli and cognitive decline in a 
cohort of older people: a prospective study. Int J Geriatr Psychiatry 2007;22:794-800.

45. Breteler MM, van Swieten JC, Bots ML, et al. Cerebral white matter lesions, vascular risk factors, and cognitive function in a population-based study: the Rotterdam Study. Neurology 1994;44:1246-52.

46. Takasugi J, Miwa K, Watanabe Y, et al. Cortical Cerebral Microinfarcts on 3T Magnetic Resonance Imaging in Patients With Carotid Artery Stenosis. Stroke 2019;50:639-44.

47. Iadecola C. The pathobiology of vascular dementia. Neuron 2013;80:844-66.

48. Pini L, Pievani M, Bocchetta M, et al. Brain atrophy in Alzheimer's Disease and aging. Ageing Res Rev 2016;30:25-48.

49. Carrera E, Lee LK, Giannopoulos S, et al. Cerebrovascular reactivity and cerebral autoregulation in normal subjects. J Neurol Sci 2009;285:191-4.

50. del Zoppo GJ. The neurovascular unit in the setting of stroke. J Intern Med 2010;267:156-71.

51. Viticchi G, Falsetti L, Buratti L, et al. Metabolic syndrome and cerebrovascular impairment in Alzheimer's disease. Int J Geriatr Psychiatry 2015;30:1164-70.

52. Silvestrini M, Viticchi G, Falsetti L, et al. The role of carotid atherosclerosis in Alzheimer's disease progression. J Alzheimers Dis 2011;25:719-26.

53. Balucani C, Viticchi G, Falsetti L, et al. Cerebral hemodynamics and cognitive performance in bilateral asymptomatic carotid stenosis. Neurology 2012;79:1788-95.

54. Chen WH, Jin W, Lyu PY, et al. Carotid Atherosclerosis and Cognitive Impairment in Nonstroke Patients. Chin Med J 2017;130:2375-9.

55. Yew B, Nation DA; Alzheimer's disease neuroimaging initiative. Cerebrovascular resistance: effects on cognitive decline, cortical atrophy, and progression to dementia. Brain 2017;140:1987-2001.

56. Turk M, Pretnar-Oblak J, Zupan M, et al. Ultrasound diagnosis of carotid artery stiffness in patients with ischemic leukoaraiosis. Ultrasound Med Biol 2015;41:64-71.

57. Rocque BG, Jackson D, Varghese T, et al. Impaired cognitive function in patients with atherosclerotic carotid stenosis and correlation with ultrasound strain measurements. J Neurol Sci 2012;322:20-4.

58. Cagnin A, Kassiou M, Meikle SR, et al. In vivo evidence for microglial activation in neurodegenerative dementia. Acta Neurol Scand Suppl 2006;185:107-14.
59. Arntzen KA, Schirmer H, Johnsen SH, et al. Carotid atherosclerosis predicts lower cognitive test results: a 7-year follow-up study of 4,371 stroke-free subjects - the Tromsø study Cerebrovasc Dis 2012;33:159-65.

60. Martinić-Popović I, Lovrencic-Huzjan A, Demarin V. Assessment of subtle cognitive impairment in strokefree patients with carotid disease. Acta Clin Croat 2009;48:231-40.

61. Wang T, Mei B, Zhang J. Atherosclerotic carotid stenosis and cognitive function. Clin Neurol Neurosurg 2016;146:64-70.

62. Alexandrov AV. The Spencer's Curve: clinical implications of a classic hemodynamic model. J Neuroimaging 2007;17:6-10.

63. Rossetti HC, Weiner M, Hynan LS, et al. Subclinical atherosclerosis and subsequent cognitive function. Atherosclerosis 2015;241:36-41.

64. Silvestrini M, Paolino I, Vernieri F, et al. Cerebral hemodynamics and cognitive performance in patients with asymptomatic carotid stenosis. Neurology 2009;72:1062-8.

65. Xiao F, Wang T, Gao L, et al. Frequency-Dependent Changes of the Resting BOLD Signals Predicts Cognitive Deficits in Asymptomatic Carotid Artery Stenosis. Front Neurosci 2018;12:416.

66. Luzzi S, Vella L, Bartolini M, et al. Atherosclerosis in the evolution of Alzheimer's disease: can treatment reduce cognitive decline? J Alzheimers Dis 2010;20:893-901.

67. Naylor AR, Ricco JB, de Borst GJ, et al. Editor's Choice - Management of Atherosclerotic Carotid and Vertebral Artery Disease: 2017 Clinical Practice Guidelines of the European Society for Vascular Surgery (ESVS). Eur J Vasc Endovasc Surg 2018;55:3-81.

68. Demarin V, Zavoreo I, Kes VB. Carotid artery disease and cognitive impairment. J Neurol Sci 2012;322:107-11.

69. Xia ZY, Sun QJ, Yang H, et al. Effect of Carotid Artery Stenting on Cognitive Function in Patients with Internal Carotid Artery Stenosis and Cerebral Lacunar Infarction: A 3-Year Follow-Up Study in China. PLoS One 2015;10:e0129917.

70. Nanba T, Ogasawara K, Nishimoto H, et al. Postoperative cerebral white matter damage associated with cerebral hyperperfusion and cognitive impairment after carotid endarterectomy: a diffusion tensor magnetic resonance imaging study. Cerebrovasc Dis 2012;34:358-67.

71. Ghogawala Z, Amin-Hanjani S, Curran J, et al. The effect of carotid endarterectomy on cerebral blood flow and cognitive function. J Stroke Cerebrovasc Dis 2013;22:1029-37. 
Page 10 of 10

72. Lattanzi S, Carbonari L, Pagliariccio G, et al. Neurocognitive functioning and cerebrovascular reactivity after carotid endarterectomy. Neurology 2018;90:e307-15.

73. Marshall RS, Pavol MA, Cheung YK, et al. Cognitive Impairment Correlates Linearly with Mean Flow Velocity by Transcranial Doppler below a Definable Threshold. Cerebrovasc Dis Extra 2020;10:21-7.

Cite this article as: Viticchi G, Falsetti L, Potente E, Bartolini M, Silvestrini M. Impact of carotid stenosis on cerebral hemodynamic failure and cognitive impairment progression: a narrative review. Ann Transl Med 2021;9(14):1209. doi: 10.21037/atm-20-7226
Viticchi et al. Carotid stenosis and cognitive impairment progression

74. Balestrini S, Perozzi C, Altamura C, et al. Severe carotid stenosis and impaired cerebral hemodynamics can influence cognitive deterioration. Neurology 2013;80:2145-50.

75. Howard VJ, Meschia JF, Lal BK, et al. Carotid revascularization and medical management for asymptomatic carotid stenosis: Protocol of the CREST-2 clinical trials. Int J Stroke 2017;12:770-8. 